Textures and Microstructures, 1988, Vol. 7, pp. 317-337

Reprints available directly from the publisher

Photocopying permitted by license only

C) 1988 Gordon and Breach Science Publishers Inc.

Printed in the United Kingdom

\title{
Development of the Rolling Texture in Titanium
}

\author{
H. P. LEE, $†$ C. ESLING $\ddagger$ and H. J. BUNGE $\dagger$ \\ † Department of Physical Metallurgy TU Clausthal FRG \\ ¥ Laboratoire de Métallurgie des Materiaux Polycristallins, University of Metz, \\ France \\ $\S$ Now: China Steel Corp. Hsiao Kang, Taiwan
}

(Received September 14, 1987)

\begin{abstract}
The complete ODF of titanium, cold rolled up to $80 \%$ deformation, was calculated using the series expansion method, including the zero range method. The rolling texture obtained after $80 \%$ deformation is mainly characterized by the well-known orientation $\{0001\}\langle 1010\rangle \pm 40^{\circ} \mathrm{TD}$ but with distinct spread ranges about it. At about $40 \%$ deformation several other texture components are found of which the component $\{0001\}\langle 11 \overline{2} 0\rangle$ must be mentioned. Further features of the obtained textures are a minor component as well as characteristic zero ranges. Texture development as function of the rolling degree can be divided into three ranges judged by increase or decrease of various texture components. In the early stages twinning in two different types of twinning systems is assumed whereas at higher deformation degrees the formation of the rolling texture is ascribed to glide deformation only.
\end{abstract}

KEY WORDS: Titanium, cold rolling, ODF analysis, glide, twinning.

\section{INTRODUCTION}

The rolling texture of titanium was first studied by Williams and Eppelsheimer (1953) and later on by several other authors (see e.g. Wassermann and Grewen 1969). Its main features are generally described by the two-fold ideal orientation $\{0001\}\langle 10 \overline{1} 0\rangle \pm 40^{\circ} \mathrm{TD}$ which means that the hexagonal $c$-axes [0001] are tilted $\pm 40^{\circ}$ from the sheet normal direction towards the transverse direction, and the direction $\langle 10 \overline{1} 0\rangle$ is parallel to the rolling direction. This fits quite 
well into the general scheme of rolling textures in hexagonal metals. The rolling texture of subnormal metals is generally described as above, that of supernormal metals exhibits a tilt of $c$-axes towards rolling direction, and in the normal metals the $c$-axes are concentrated in normal direction.

Although there is general consent, that the ideal orientation mentioned above describes the main features of the rolling texture of titanium at higher deformation degrees sufficiently well, several other texture components have also been observed, depending on parameters such as rolling degree, temperature, purity of the material and others. A comprehensive survey was given by Zarkades and Larson (1971). They classified the texture types of titanium according to (0002) and (1010) pole figures. Detailed features of the texture can be distinguished by considering not only the intensity peak positions but also the whole distribution of intensities, mainly in the (0002) pole figure. This reveals a complex intensity spread in the (0002) pole figure in the whole range of the ND-TD-plane and also other minor intensity ranges e.g. in the vicinity of the rolling direction.

It is evident that orientation distributions like that are difficult to estimate by the (0002) pole figure alone. Rather, it is necessary to measure further pole figures in order to discriminate orientations rotated about the hexagonal $c$-axis. A thorough treatment of this problem is only possible by calculating the orientation distribution function starting from several experimentally measured pole figures. ODF-analysis of a hexagonal metal was first carried out by Morris and Heckler (1969) using recrystallized titanium sheet. ODFanalysis of cold rolled titanium was done by Dervin et al. (1977), Naka et al. (1977), Knight (1977) and Davies et al. (1978). In these investigations the ODF was calculated using a series expansion method based on even terms only which can be determined directly from pole figures.

As was shown, however, by Matthies (1979) the complete ODF must include the odd coefficients, too. These terms are extinguished in the pole figures because of the $(h k i l)-(\bar{h} \bar{k} \overline{i l})$ superposition. If the texture contains zero-ranges, as is often the case, also odd coefficients can be calculated-and hence the complete ODF-using the zero-range method (Bunge, Esling 1979). In order to study the rolling texture of titanium in more detail, distinguishing also minor 
orientation components, it is thus necessary to calculate the complete ODF including the odd terms of the series expansion.

It is the purpose of this paper to study the development of the rolling texture of titanium as a function of rolling degree by determining complete orientation distribution functions.

\section{EXPERIMENTAL PROCEDURE}

The material used was commercially pure titanium of grade 1 (low oxygen). The chemical composition is given in Table 1. The material was obtained in the form of hot rolled sheet, $3.7 \mathrm{~mm}$ thick. It was recrystallized with a mean grain size of $17 \mu \mathrm{m}$. It exhibited a hot rolling texture as shown in Figures 2 and 7. The material was then cold rolled in the former hot rolling direction without lubrication using a laboratory two-roll mill with $180 \mathrm{~mm}$ diameter to a total of $80 \%$ reduction of area.

Samples for X-ray texture measurement were taken at intermediate degrees of deformation. Complete pole figures were obtained by the oblique section method described by Elias and Heckler (1967) and later on by Welch (1980). Strips cut at $45^{\circ}$ to the rolling direction were assembled as shown in Figure 1. The final sample surface is thus perpendicular to a "pseudo $\langle 111\rangle$ " direction. It represents the average of all four quadrants of the pole figure as well as the cross section of the sheet. Before assembling, the strips were chemically degreased. Reflection pole figures were then measured using an automated texture goniometer ATEMA-C (Puch, Klein, Bunge 1984) and $\mathrm{Cuk}_{\alpha}$-radiation up to a limiting angle of $\alpha=70^{\circ}$ and in steps of $5^{\circ} \times 3.6^{\circ}$ in $\alpha$ and $\beta$ respectively. The so obtained pole figures were transformed into the normal coordinate system RD, TD, ND by a transformation program using linear interpolation between the original measuring steps. The pole figures (1010) (0002) (1011) (1120) were thus obtained. Two of them, for each deformation degree, are shown in Figure 2. In this

Table 1 Composition of titanium sheet

\begin{tabular}{lllllll}
\hline $\mathrm{Ni}$ & $\mathrm{Mn}$ & $\mathrm{Fe}$ & $\mathrm{C}$ & $\mathrm{N}$ & $\mathrm{O}$ & $\mathrm{Ti}$ \\
\hline 0.015 & 0.004 & 0.038 & 0.0075 & $<0.01$ & 0.05 & balance \\
\hline
\end{tabular}



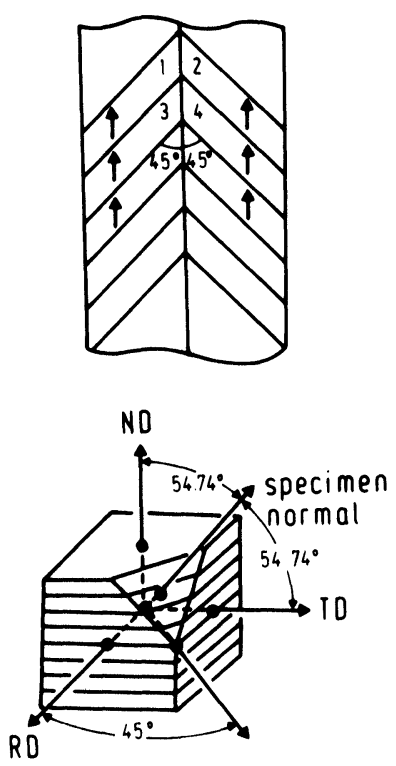
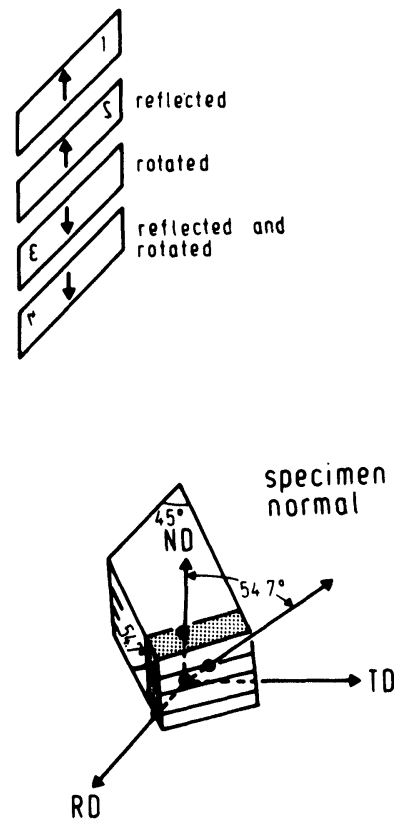

Figure 1 Sample preparation using the oblique section method.

figure also the zero-ranges are indicated which are needed, later on, in order to determine the odd part of the series expansion.

The (10i1) and (0002) pole figures are slightly overlapped in $2 \vartheta$. This effect increases due to increasing defocalization with increasing tilt angle $\alpha$. Since the tilt angle was limited to $\alpha_{\max }=70^{\circ}$, extreme values of overlapping were not reached. Hence, the effect could be avoided by measuring these two pole figures slightly off the Bragg-position at the non-overlapping side of each of the peaks.

Using neutron diffraction and a spherical sample (Tobisch, Bunge 1972) no defocalization occurs. The overlapping is much smaller in this case and can easily be avoided. Hence, some of the measurements were repeated using neutron diffraction at the research reactor at Jülich (Will 1985). The results shown in Figure 3 are very similar to those obtained by $\mathrm{X}$-ray diffraction. Hence, $\mathrm{X}$-ray pole figures could be assumed to be essentially free of overlap and could be used in the following as the starting data for pole figure inversion. 

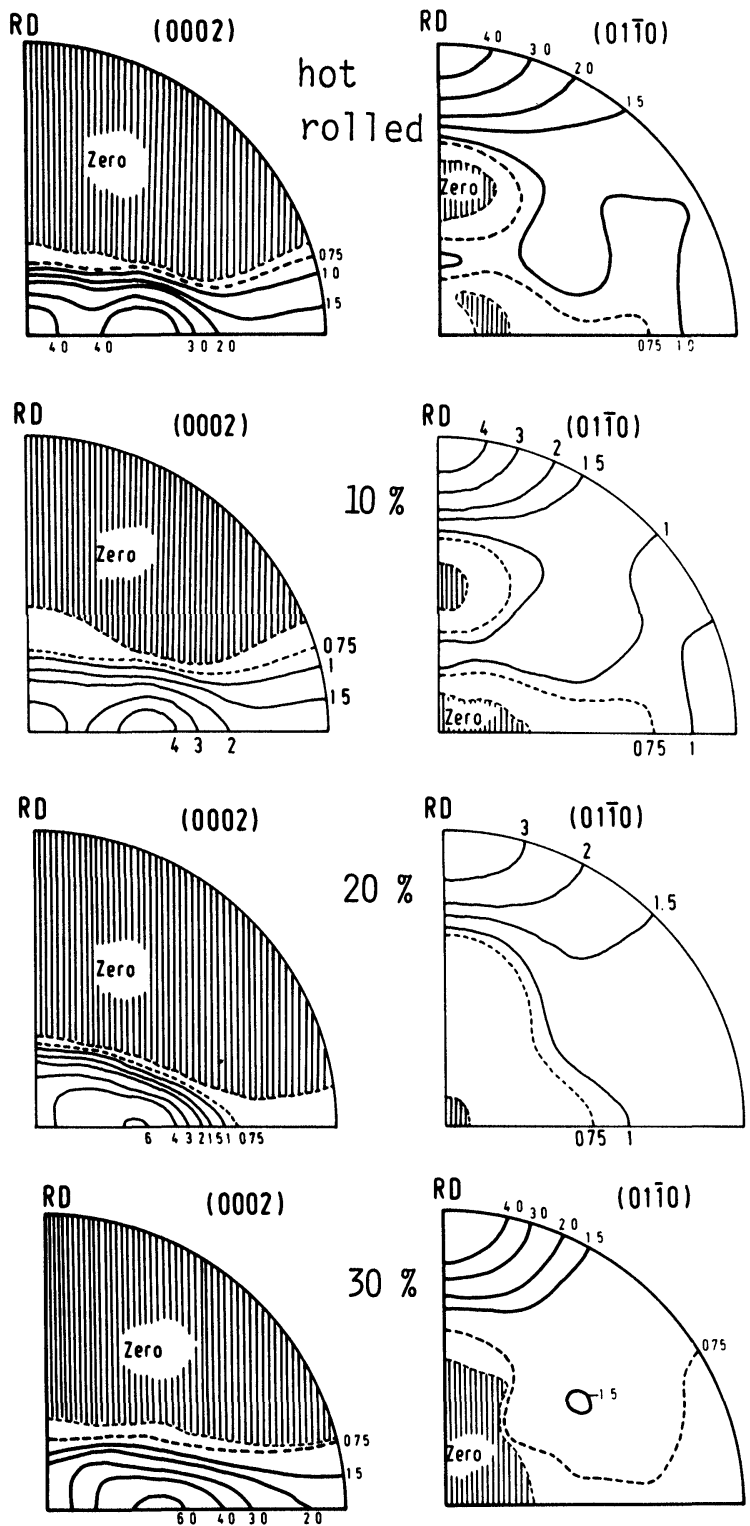

Figure 2 Pole Figures (0002) and (0110) of titanium hot rolled and cold rolled at different degrees of deformation measured by X-ray diffraction. 

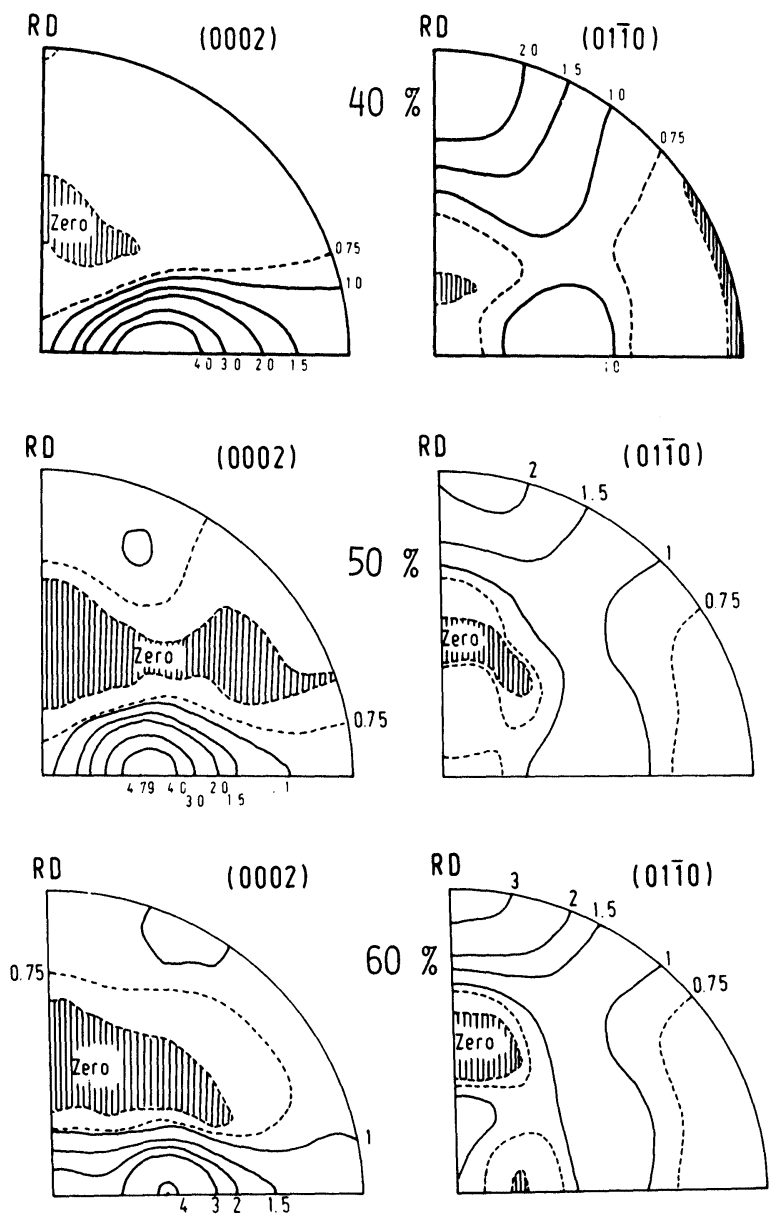

Figure 2 (contd.)
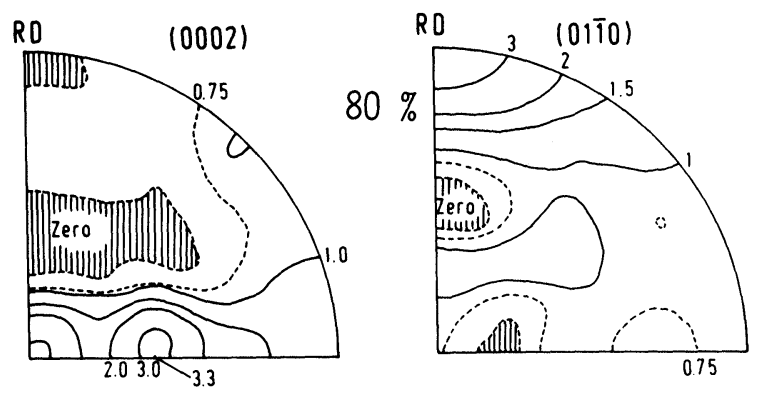

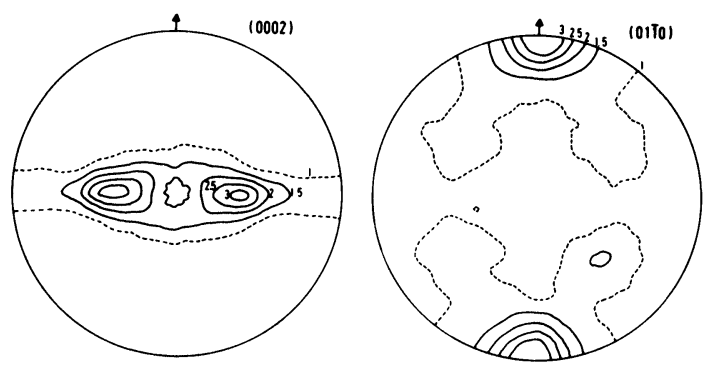

Figure 3 Pole figures $(0002)$ and $(01 \overline{1} 0)$ of titanium cold rolled $60 \%$ measured by neutron diffraction.

\section{POLE FIGURE INVERSION}

In order to calculate orientation distribution functions, ODF, from pole figures, the series expansion method was used (e.g. Bunge 1969,1982 ). With four figures available, a maximum degree of $l=22$ can be obtained. In order to level out the unavoidable errors in the pole figures it is, however, indicated to use at least one pole figure more than the absolute minimum. Then a maximum degree of $l=16$ is possible with four pole figures. As will be seen later this degree was sufficient (judged by the convergence of the coefficients).

In order to define the orientation $g$ by Euler angles, an orthogonal crystal coordinate system $K_{B}$ must be chosen with respect to the hexagonal crystal lattice. This was done in such a way that the $x_{1}$ direction of $K_{B}$ was parallel to the crystal $\langle 10 \overline{10}\rangle$ direction. (It must be mentioned that also another choice is possible with $x_{1} \|\langle 11 \overline{2} 0\rangle$. Together with the ambiguity of Euler angles according to the definitions used by Bunge (1965) and Roe (1965) respectively, this leads to a total of four possible choices of Euler angles in the case of hexagonal-orthorhombic symmetry.)

The odd coefficients of the series expansion cannot be deduced directly from the series expansion of the pole figures since the latter ones are superpositions of the (hkil) and $(\bar{h} \bar{k} \overline{i l})$ pole figures (Matthies 1979). Odd coefficients can, however, be determined using the zero-range method (Bunge and Esling 1979). In experimental measurements it is often difficult to fix exactly the borderline of the 
zero range. In fact, the value "zero" can only be defined within the limits of experimental accuracy. Hence, some model calculations were necessary in order to find an optimum choice of the zero range. If the zero range is chosen too small then it is insufficient to determine the odd coefficients. If it is chosen too large then it includes ranges which are, in fact, different from zero. In both cases errors are introduced of which the minimum has to be found. (For details see: Lee et al. 1984a, b, 1986)

To this purpose a computer program of the zero-range method for hexagonal crystal symmetry was worked out (Lee 1985). It was checked by a theoretical texture similar to the experimental texture of $60 \%$ deformation i.e. consisting of the ideal orientation $B=$ $\{0001\}\langle 10 \overline{10}\rangle \pm 40^{\circ} \mathrm{TD}$ with the Euler angles $g=\left\{0^{\circ}, 40^{\circ}, 0^{\circ}\right\}$ and a Gaussian spread of $\omega=18^{\circ}$. In this case the texture coefficients (even and odd) can be calculated theoretically. Hence, the effectiveness of the zero-range program can easily be checked. In Figure 4 the mean absolute values of the coefficients are shown. The differences between the theoretical values and the ones obtained by the zero-range method are within the drawing accuracy. A section of the ODF at $\varphi_{1}=\varphi_{2}=0$ is shown in Figure 5. Also here the theoretical curve is identical with the one obtained by the zero-

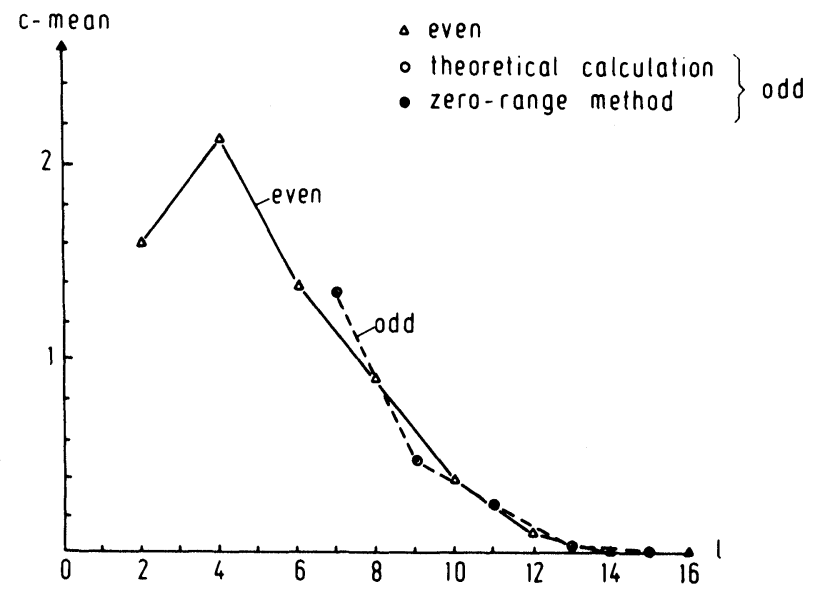

Figure 4 Mean values of the coefficient $C_{l}^{\mu \nu}$ over $\mu$ and $v$ of a theoretical texture. The coefficients obtained by the zero-range method are nearly identical with the theoretical ones. 


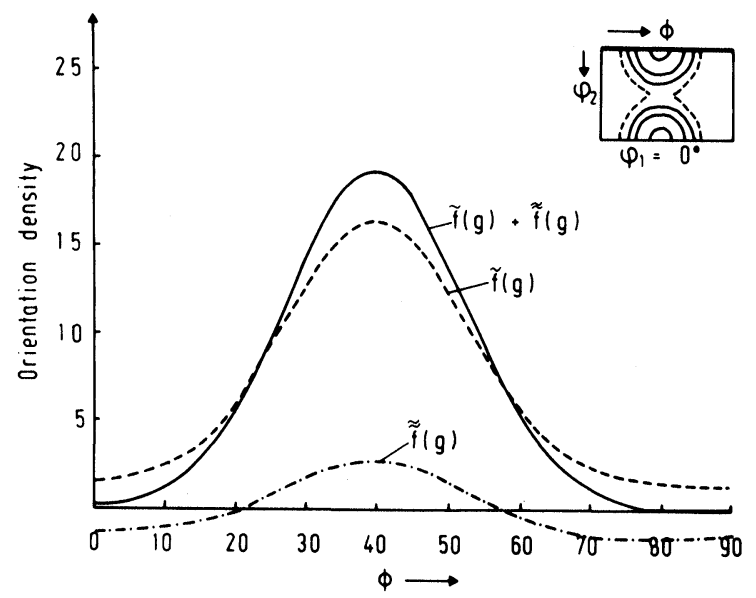

Figure 5 Section through the theoretical ODF. The full line, representing the complete ODF obtained by the zero-range method is indistinguishable from the theoretical one.

range method. Hence, the program could be applied to the experimental pole figures.

\section{ODF-ANALYSIS}

Starting with the pole figures of Figure 2, ODF-analysis was carried out with a series expansion up to $l=16$ and $l_{1}=11$ respectively for the even and odd part (Lee, Bunge 1984). This degree is sufficient compared with the convergence of the coefficients which can be seen in Figure 6 showing the mean absolute values of the $C_{l}^{\mu v}$ over $\mu$ and $v$. The orientation distribution functions $f(g)$ are shown in Figure 7. The influence of the odd part is generally not very strong as is to be seen in Figure 8 which shows a section along $\varphi_{1}=\varphi_{2}=0^{\circ}$ through the orientation space. Thus, omission of the odd part does not change the main texture components essentially, it does, however, change their relative intensities, and minor components in the range of the random orientation density may be essentially modified.

The main features of the texture are contained in the section $\varphi_{1}=0^{\circ}$ as is shown in Figure 9. The main orientations may be 

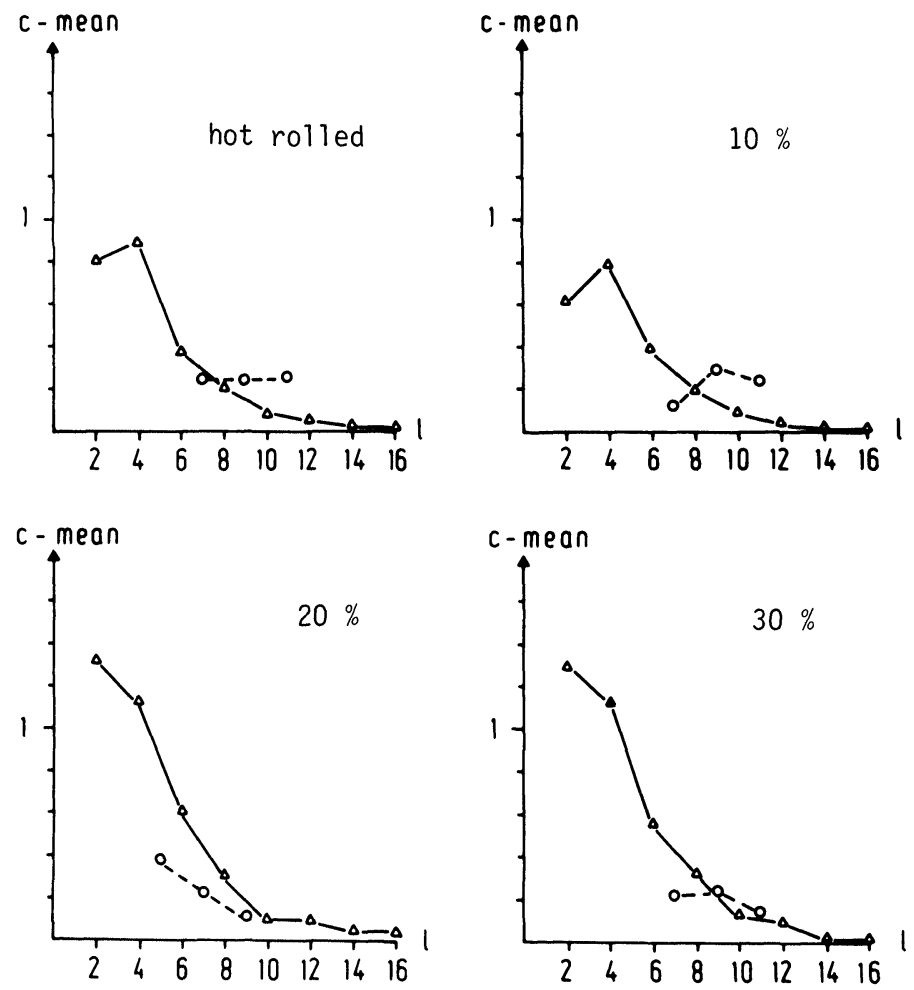

Figure 6 Mean values of the coefficient $C_{l}^{\mu v}$ over $\mu$ and $v$ for titanium cold rolled to different degrees of deformation. $\Delta$ even coefficients, $O$ odd coefficents (zero-range method).

indexed as given in Table 2. Besides the main components in the section $\varphi_{1}=0^{\circ}$, a minor component $M$ is found at lower deformation degrees. Another feature of the textures is the occurrence of a zero range $Z$ in the section $\varphi_{1}=0^{\circ}$, centered at the orientation $\{10 \overline{1} 0\}\langle 11 \overline{2} 0\rangle$. Furthermore, large zero ranges are observed in the sections $\varphi_{1} \neq 0$.

Between the main orientations, spread ranges are extended. The orientation densities along the three main spread ranges $A-B-C$, $A-D$ and $B-E$ are shown in Figure 10 .

Finally, the texture development with the degree of deformation 

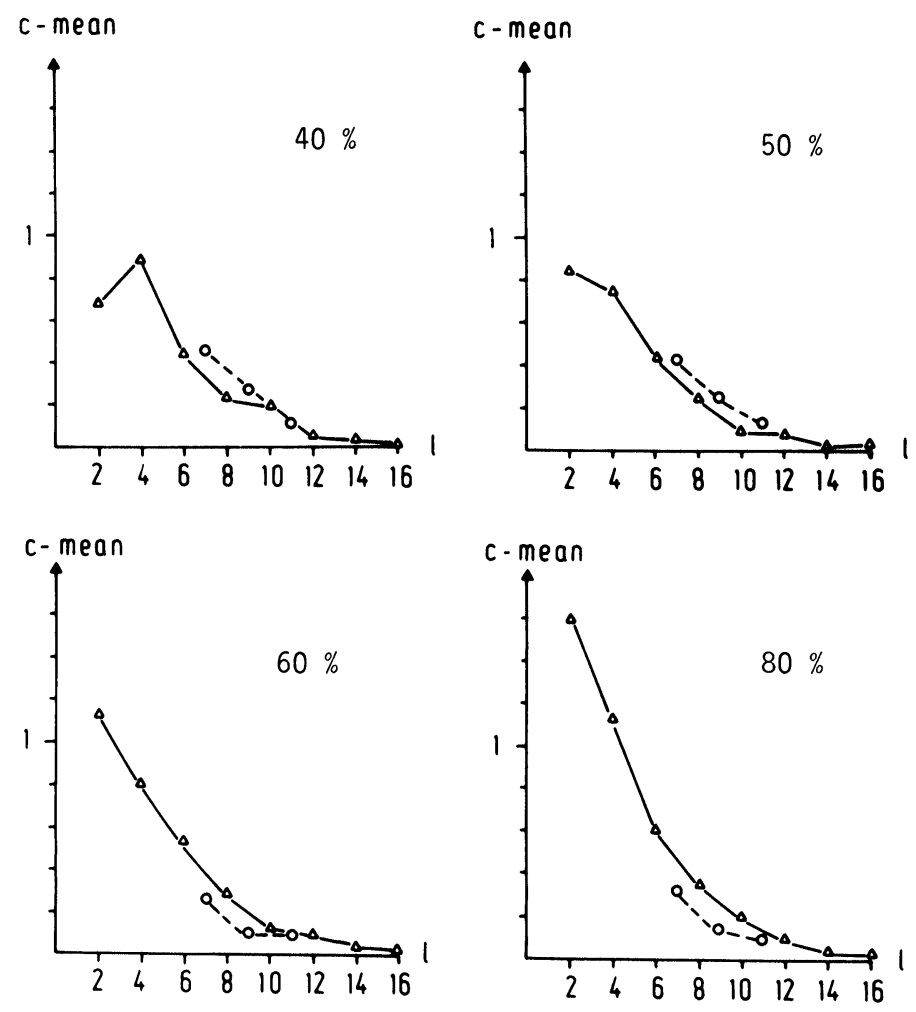

Figure 6 (contd.)

can be summarized in Figure 11 which shows the dependence of intensities in the points $A-E$ on the deformation degree.

Considering Figures 9 and 11, the main features of the texture development can be summarized as follows:

- The hot rolling texture is dominated by an orientation range extended between the orientations $B$ and $E$, having the [0001]-direction at $\pm 40^{\circ}$ from ND towards $T D$, with free rotation about this axis.

- During cold rolling, this range of orientations splits up with the orientation $B$ steadily increasing and the orientation $E$ decreasing at first. Later on the development of this orientation (on a lower level) is somewhat irregular. 

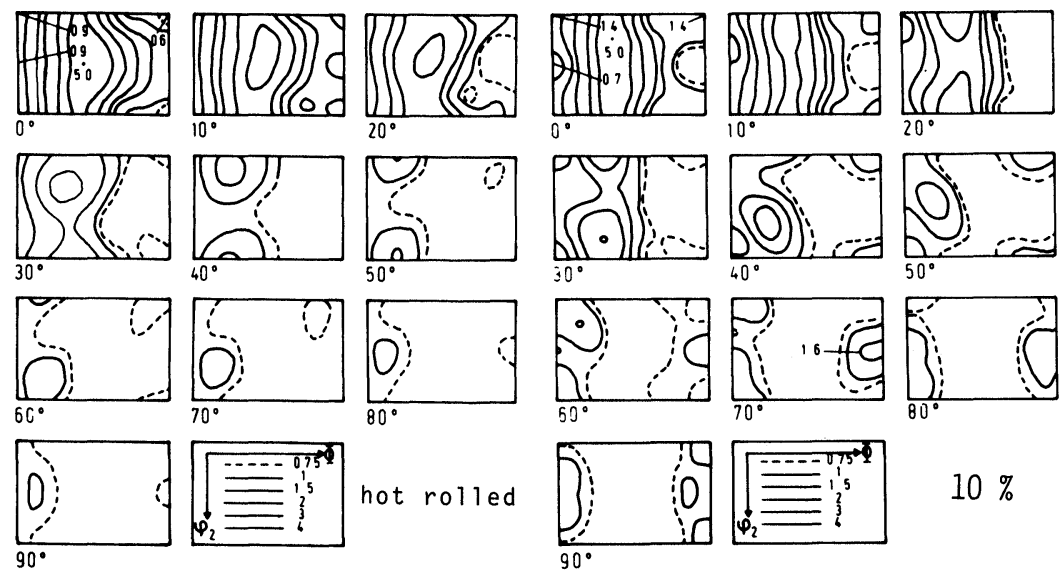

$10 \%$
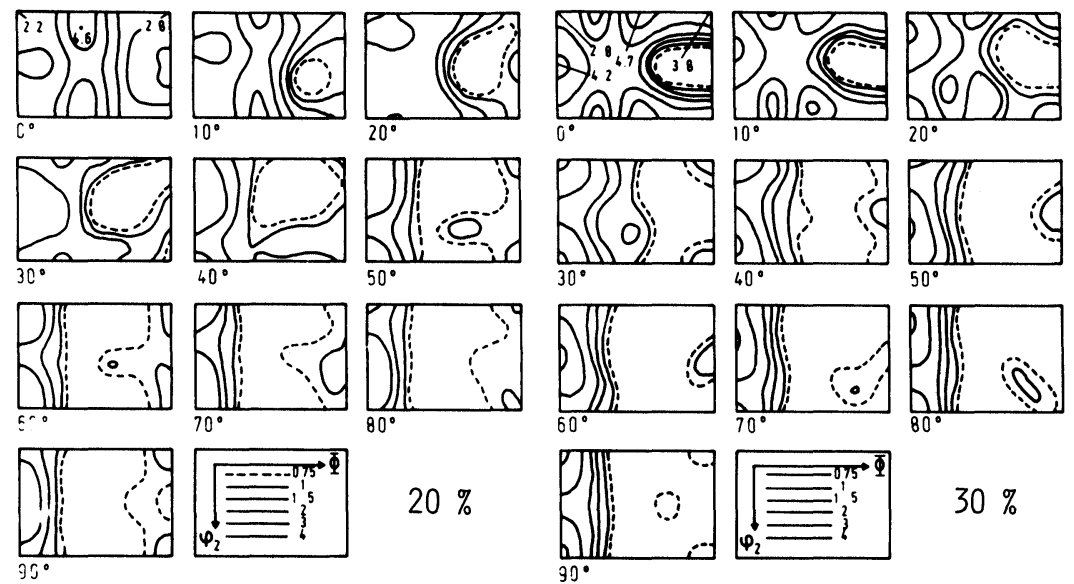

$20 \%$

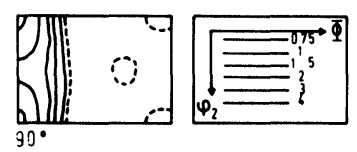

$30 \%$

Figure 7 Complete orientation distribution functions of titanium cold rolled to different degrees of deformation

- The orientation $D$ increases from a low level at the beginning, runs through a rather high intermediate level and then decreases again to a low level.

- Similar to this development is the development of the zero range in the $\varphi_{1}=0^{\circ}$-section. It is very pronounced at intermediate deformation degrees where orientation $D$ takes on its highest values. 

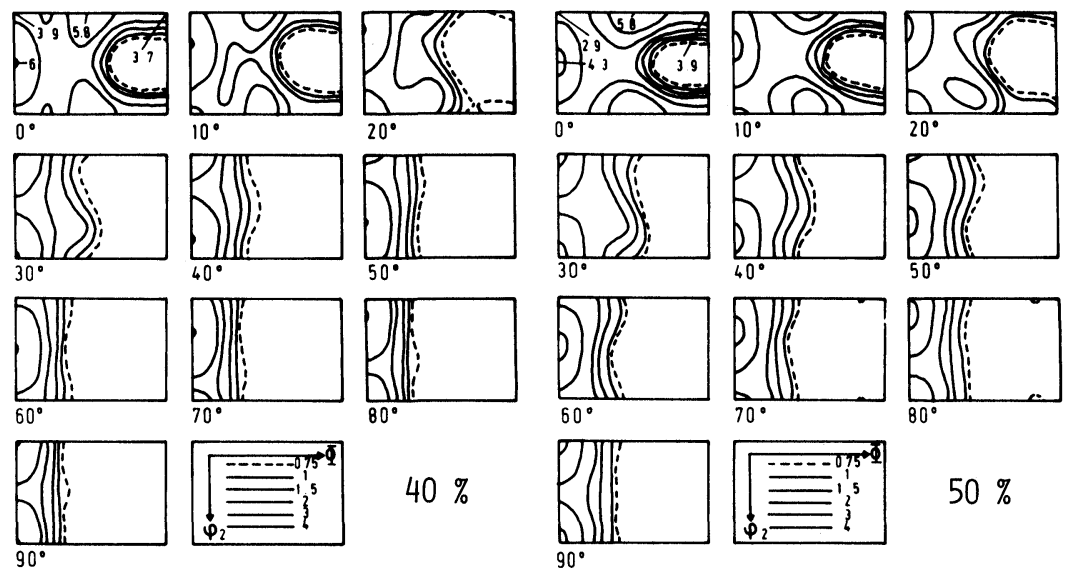

$40 \%$
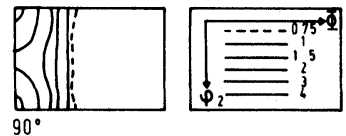

$50 \%$
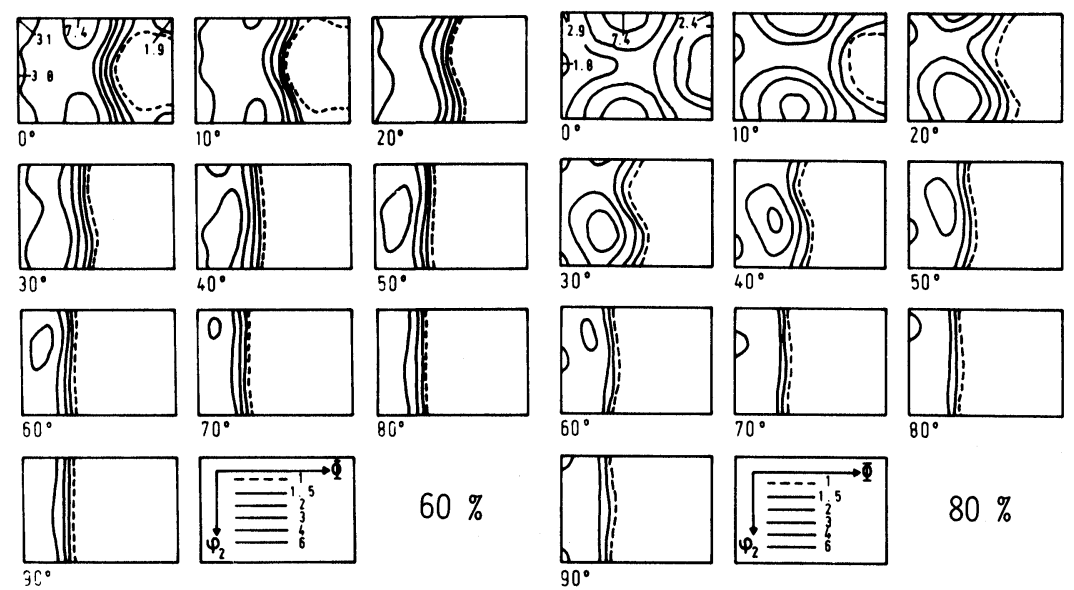

$60 \%$
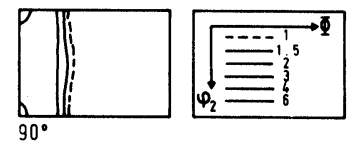

$80 \%$

Figure 7 (contd.)

- An intermediate increase, though smaller than that of the component $D$, is also observed in the components $A$ and $C$, which decrease slowly to intermediate levels at $80 \%$ deformation. At this deformation, also the pronounced zero range observed at intermediate deformations, has slightly been filled up again.

Besides this general picture of texture development, two details must be mentioned which do not fit very well into the general lines. 


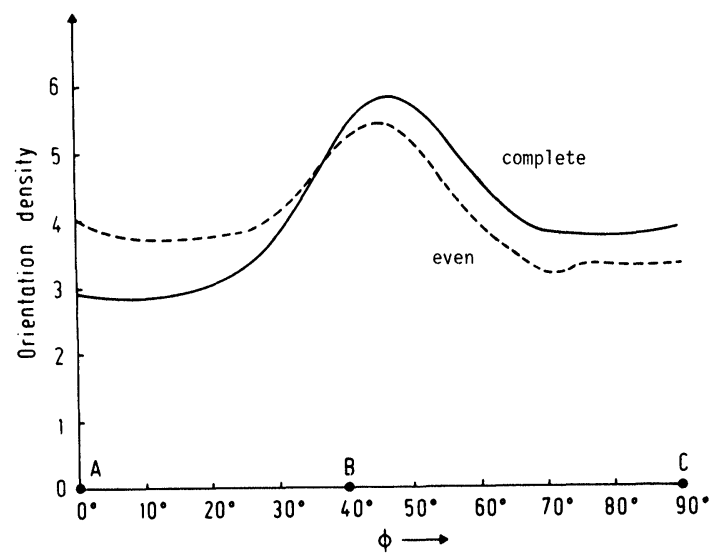

Figure 8 Section at $\varphi_{1}=\varphi_{2}=0^{\circ}$ of the ODF of titanium cold rolled $40 \%$ showing the contribution of the odd terms.

These are the relatively high value of component $E$ at $60 \%$ deformation and the split of component $B$ at $30 \%$ deformation. These two points need further investigations. The same holds for the development of the components $A, C, E$ at higher deformation degrees. From Figure 11 it may be concluded that these components converge to a low intensity level but do not decrease to zero. Component $B$ seems to increase continuously whereas component $D$ seems to decrease continuously at higher deformation degrees. Assuming this picture, the experimental results may be summarized and idealized as is shown in Figure 12 which exhibits the shematic development of the main and minor components as a function of the degree of deformation.

\section{DISCUSSION}

The development of the cold rolling texture of titanium is summarized in Figure 12 showing schematically the orientation densities in the texture components as a function of the degree of deformation. The starting hot rolling texture contains essentially the orientations $B$ and $E$ having the [0001]-direction about $40^{\circ}$ from ND towards TD whereby all orientations obtained by rotation about 

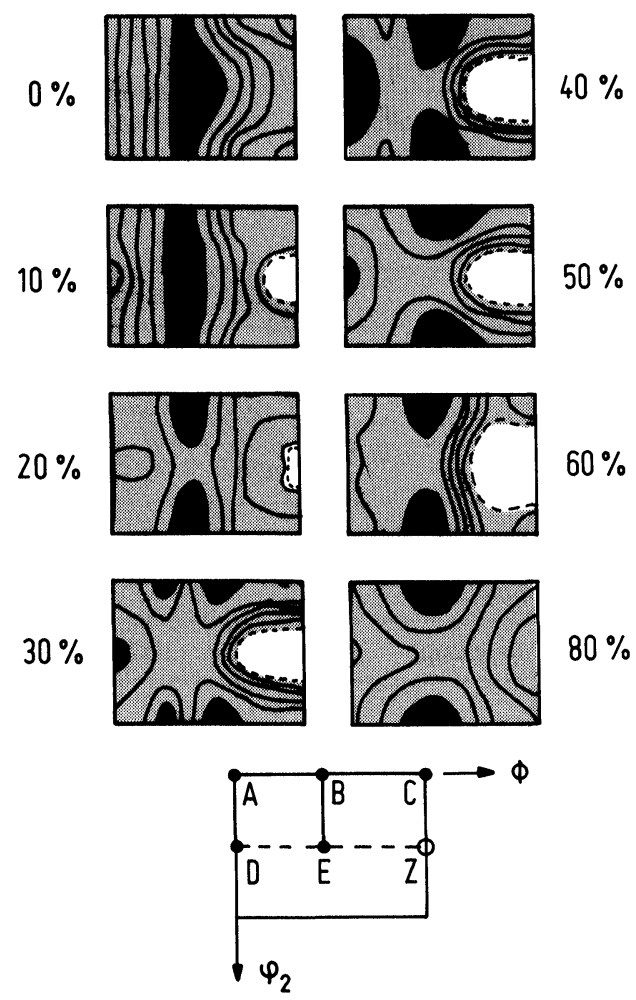

Figure 9 The sections $\varphi_{1}=0^{\circ}$ of the ODF for different degrees of deformation containing the main orientations $A$ to $E$ and the zero-range $Z$, the indices of which are given in Table 2.

[0001] are nearly equally frequent. This texture is assumed to be due to primary recrystallization and grain growth as studied by Nauer-Gerhardt and Bunge (1984). In this paper the texture immediately after primary recrystallization was found to be mainly the orientation $B=\{0001\}\langle 1010\rangle \pm 40^{\circ} \mathrm{TD}$ which is thus identical with the rolling texture. During continuous grain growth following primary recrystallization, this orientation was replaced with the orientation $E=\{0001\}\langle 11 \overline{2} 0\rangle \pm 40^{\circ} \mathrm{TD}$ which is thus the grain growth texture. The latter one is rotated $30^{\circ}$ about the hexagonal $c$-axis [0001]. After grain growth by a factor of 10 in the mean grain 
Table 2 Texture components observed in titanium (setting of the crystal coordinate system $K_{B}$ with $x_{1} \|\langle 10 \overline{0} 0\rangle$ )

\begin{tabular}{lllll}
\hline & Low-index representation & \multicolumn{3}{l}{ Euler angles } \\
\hline & \multicolumn{1}{l}{$\varphi_{1}$} & $\phi$ & $\varphi_{2}$ \\
\hline Main components & $\{0001\}\langle 10 \overline{1} 0\rangle$ & $0^{\circ}$ & $0^{\circ}$ & $0^{\circ}$ \\
\hline $\mathrm{B}$ & $\{0001\}\langle 10 \overline{1} 0\rangle \pm 40^{\circ} \mathrm{TD}$ & $0^{\circ}$ & $40^{\circ}$ & $0^{\circ}$ \\
\hline $\mathrm{C}$ & $\{11 \overline{2} 0\}\langle 10 \overline{1} 0\rangle$ & $0^{\circ}$ & $90^{\circ}$ & $0^{\circ}$ \\
\hline $\mathrm{D}$ & $\{0001\}\langle 11 \overline{2} 0\rangle$ & $0^{\circ}$ & $0^{\circ}$ & $30^{\circ}$ \\
\hline $\mathrm{E}$ & $\{0001\}\langle 11 \overline{2} 0\rangle \pm 40^{\circ} \mathrm{TD}$ & $0^{\circ}$ & $40^{\circ}$ & $30^{\circ}$ \\
\hline & Minor component & & & \\
\hline $\mathrm{M}$ & (no simple indexing) & $70^{\circ}$ & $90^{\circ}$ & $30^{\circ}$ \\
\hline & Zero range & & & \\
\hline $\mathrm{Z}$ & $\{10 \overline{1} 0\}\langle 11 \overline{2} 0\rangle$ & $0^{\circ}$ & $90^{\circ}$ & $30^{\circ}$ \\
\hline
\end{tabular}

size the original recrystallization component $B$ had nearly completely disappeared. In the present hot-rolling texture both components appear with nearly equal intensity. Hence, it must be assumed that a certain amount of grain growth had already occurred after primary recrystallization.

The rolling texture at $80 \%$ deformation is dominated by the component $B=\{0001\}\langle 10 \overline{1} 0\rangle \pm 40^{\circ} \mathrm{TD}$ which is commonly assumed to be the end-oientation in the titanium rolling texture. There are, however, several other components present in this texture $(A, C$, $E)$ although with much lesser intensities. The main component $B$ increases steadily during rolling and this increase seems to be continued at higher deformation degrees than $80 \%$.

According to the schematic Figure 12, the development of the rolling texture of titanium may be divided into 2 major ranges, i.e. those lower and higher than $40 \%$ respectively. Below $40 \%$ the intermediate component $D$ increases and above $40 \%$ it decreases again, and finally it disappears. Above $60 \%$ all observed texture changes are rather small. If also the minor component $M$ is taken 

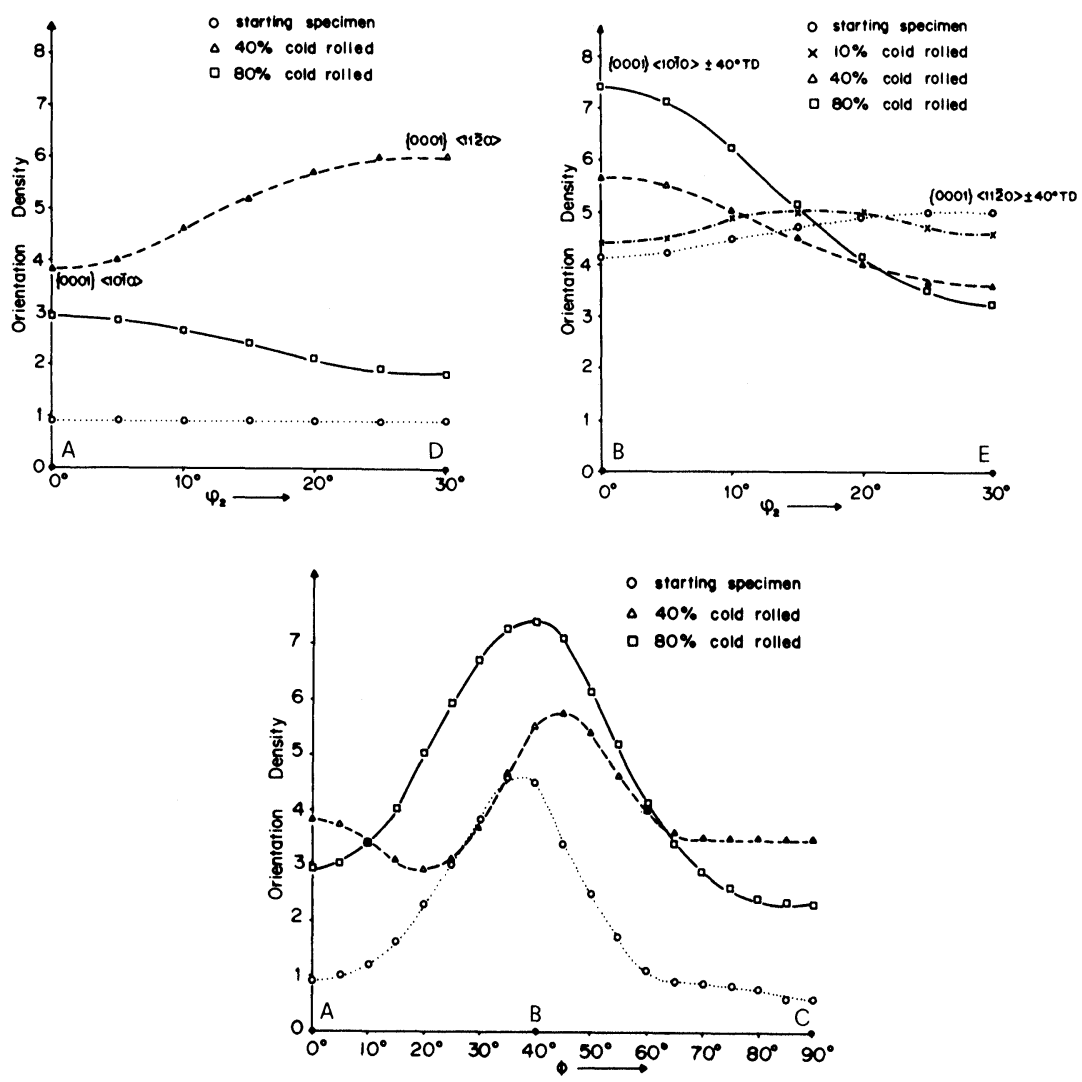

Figure 10 Sections of some of the ODF of Figure 7 along the three main paths shown in Figure 9.

into consideration a further subdivison at about $15 \%$ can be seen, below which the minor component is being built up. Above $15 \%$ this component disappears again.

The development of the rolling texture of titanium has to be interpreted by the action of glide and twinning systems. In hexagonal metals with subnormal $c / a$-ratio the main slip system is prism slip of a-dislocations i.e. the systems $\{10 \overline{1} 0\}\langle 11 \overline{2} 0\rangle$ whereas the most important twinning systems are $\{10 \overline{1} 2\}\langle 10 \overline{1} 1\rangle$ and $\{11 \overline{2} 2\}\langle 11 \overline{2} 3\rangle$ (see e.g. Grewen 1973). Structure investigations showed that twinning was profuse in this material at lower 


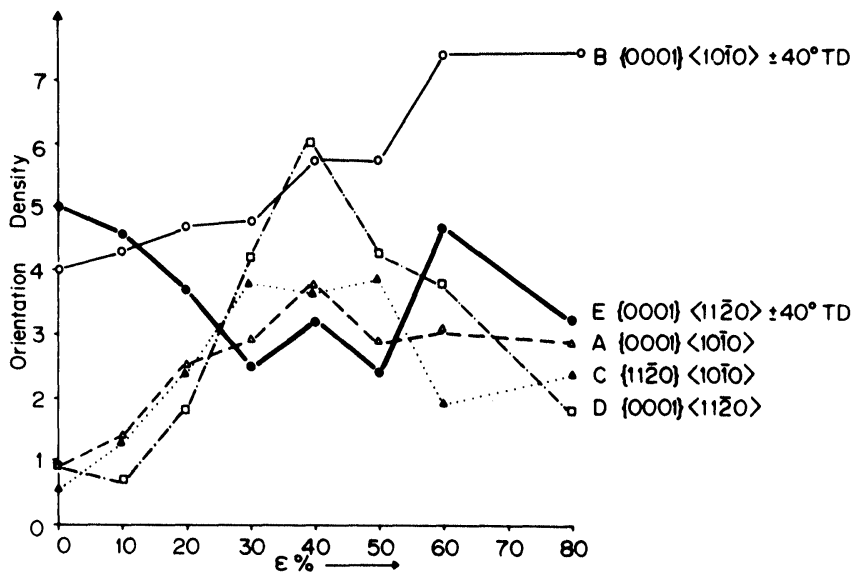

Figure 11 Orientation densities in the main orientations as a function of the degree of deformation.

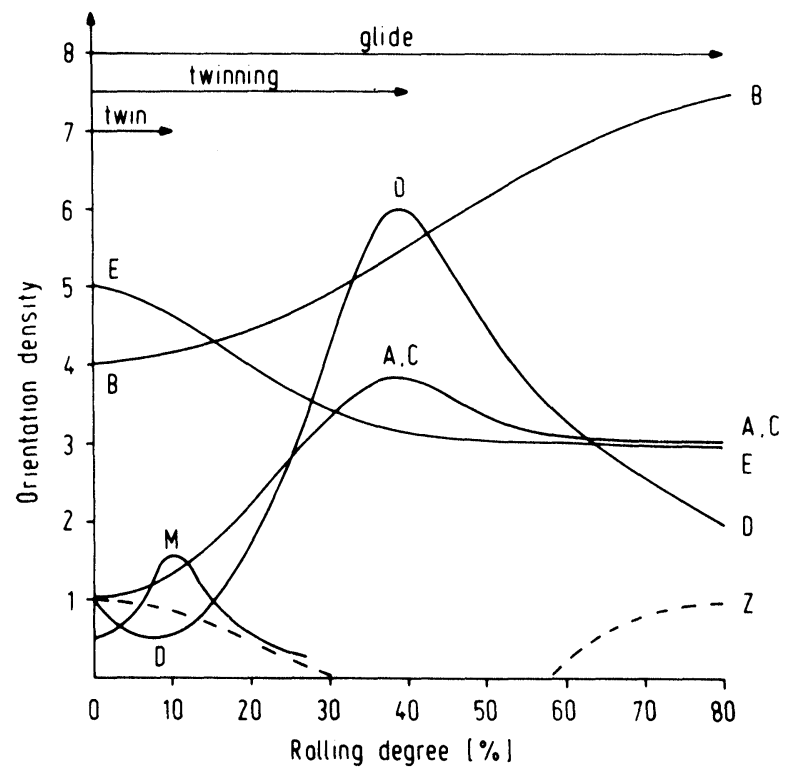

Figure 12 Schematic diagram showing the development of the rolling texture in titanium. The components $A-E$ and $M$ as well as the zero range $Z$ are explained in Table 2. 
deformation degrees (Nauer-Gerhardt, Bunge). At deformation above $40 \%$, however, twinning was no longer observed. Hence, it must be assumed that the texture development beyond $40 \%$ is intirely due to slip. This texture consists mainly of the orientation $B$ with spread ranges in the directions $A, C, E$. These orientations themselves are saddle points in the orientation distribution as can be seen in Figure 10. The orientation $D$ is strongly instable with respect to glide deformation.

At lower deformation degrees profuse twinning was observed. Hence, it may be concluded that the orientation $D$ is due to twinning. Furthermore, a zero-range developed at the same time. Hence, it may be assumed that crystals oriented in this range are twinned into the orientation $D$. Since, in this case the hexagonal axis moves from a position parallel to TD into a position parallel to ND, a twinning system which leads to a shortening in this direction must be operative. Among the two abundantly observed twin systems this is the case for one of the systems of the type $\{10 \overline{1} 2\}\langle 10 \overline{1} 1\rangle$ (see e.g. Nauer-Gerhardt, Bunge). It is also in agreement with this assumption that the orientation $C$ which also has the $c$-axis parallel to TD does apparently not twin since it would bring the $c$-axis near to a direction in the plane ND-RD such that it should be elongated rather than shortened by the rolling process.

With a similar argumentation also the minor component $M$ can be assumed to be the product of twinning. This component has the $c$-axis in the vicinity of RD. Hence, it must be due to a twin system which elongates this direction. It may thus be suggested that this component is due to twinning of the orientation $D$ according to a twinning system belonging to the type $\{11 \overline{2} 2\}\langle 11 \overline{2} 3\rangle$. As is seen in Figures 9 and 12 the orientation $D$ is present at the beginning in a small amount and its density initially still decreases at the same time as the orientation $M$ increases. According to these assumptions, it must be further assumed that crystals which have atained the orientation $D$ by $\{10 \overline{1} 2\}\langle 10 \overline{1} 1\rangle$ twinning do not further twin in second generation by the system $\{11 \overline{2} 2\}\langle 11 \overline{2} 3\rangle$ into the minor component $M$. Furthermore, if the orientation $B$ is solely attributed to glide deformation, then the prism plane cannot be the only glide plane. Rather, it must be assumed that pyramidal slip of $a$-dislocations as well as of $(c+a)$-dislocations must take place.

Because of the complexity of the possible slip and twinning 
systems the above considerations can only be a rough estimation of the occurring mechanisms. More detailed information will be obtained by further analysis of the operative twinning systems which is presently being carried out (Nauer-Gerhardt, Bunge). A quantitative model calculation of the obtained textures will be based on the Taylor theory. The present results suggest, however, that this model can be restricted to prism and pyramidal glide for the higher deformation degrees.

\section{Acknowledgement}

The authors gratefully acknowledge valuable discussions with Dr. K. H. Kramer of Krupp Stahl AG who also provided the titanium sheet. The neutron diffraction measurements were carried out at KFA Jülich for which we want to thank Prof. H. Will. The work was sponsered by the German Research Foundation (DFG).

\section{References}

Bunge, H. J. (1965). Z. Metallkunde 56, 872-874.

Bunge, H. J. (1969). Mathematische Methoden der Texturanalyse. AkademieVerlag Berlin.

Bunge, H. J. (1982). Texture Analysis in Materials Science. Butterworths, London.

Bunge, H. J. and Esling, C. (1979). Journ. Phys. Lettr. 40, 627-628.

Davies, G. J., Kallend, J. S. and Knight, F. I. (1978). Textures of Materials, Proceedings of ICOTOM 5, Ed. G. Gottstein and K. Lücke, 1, 245-251.

Dervin, P., Mardon, J. P., Pernot, M., Penelle, R. and Lacombe, P. (1977). Journ. Less Common Met. 55, 25-28.

Elias, J. A. and Heckler, A. J. (1967). Trans. Met. Soc. AIME 239, 1237-1241.

Grewen, J. (1973) Proc. ICOTOM 3 Pont-à-Mousson 195-234.

Knight, F. I. (1977). Textures in titanium sheets. Thesis Cambridge.

Lee, H. P. (1985). Texturanalyse unter Anwendung der Nullbereichsmethode. Thesis, Clausthal

Lee, H. P., Esling, C. and Bunge, H. J. (1984) Proc. ICOTOM 7 Noordwijerhout 793-798.

Lee, H. P. and Bunge, H. J. (1984). Proc. 5th Internat. Conf. Titanium München 1721-1728.

Lee, H. P, Welch, P. I., Esling, C. and Bunge, H. J. (1984). Proc. ICOTOM 7, Noordwijkerhout 799-803.

Lee, H. P., Bunge, H. J. and Esling, C. (1986). Textures and Microstructures 6, 289-313.

Matthies, S. (1979). Phys. Stat. Sol. 92K, 135-138.

Morris, P. R. and Heckler, A. J. (1969). Trans. AIME 245, 1877-1881. 
Naka, S., Mardon, J. P., Dervin, P. Pernot, M., Penelle, R. and Lacombe, P. (1977). Journ. Less Common Metals 55, 277-283.

Nauer-Gerhardt, C. and Bunge, H. J. (1984). Proc. 5th Intern. Conference on Titanium München 1713-1719.

Nauer-Gerhardt, C. and Bunge, H. J. to be published.

Puch, K. H., Klein, H. and Bunge, H. J. (1984). Z. Metallkunde 75, 133-138.

Roe, R. J. (1965). J. Apply. Phys. 36, 2024-2031.

Tobisch, J. and Bunge, H. J. (1973). Texture 1, 125-127.

Wassermann, G. and Grewen, J. (1969). Texturen metallischer Werkstoffe. Springer Verlag Berlin.

Welch, P. I. (1980). Textures of Crystalline Solids 4, 99-110.

Will, G. (1985). Private communication.

Williams, D. N. and Eppelsheimer, D. S. (1953). Trans. AIME 197, 1378-1382.

Zarkades, A. and Larson, F. R. (1971). Army Materials and Mechanics Research Center TR 71-60. 